\title{
Deformed Special Relativity and Deformed Symmetries in a Canonical Framework
}

\author{
Subir Ghosh \\ Physics and Applied Mathematics Unit, \\ Indian Statistical Institute, \\ 203 B. T. Road, Calcutta 700108, India \\ And \\ Probir Pal \\ Physics Department, \\ Uluberia College, \\ Uluberia, Howrah 711315, India.
}

\begin{abstract}
:
In this paper we have studied the nature of kinematical and dynamical laws in $\kappa$-Minkowski spacetime from a new perspective: the canonical phase space approach. We discuss a particular form of $\kappa$-Minkowski phase space algebra that yields the $\kappa$-extended finite Lorentz transformations derived in [16]. This is a particular form of a Deformed Special Relativity model that admits a modified energy-momentum dispersion law as well as noncommutative $\kappa$-Minkowski phase space. We show that this system can be completely mapped to a set of phase space variables that obey canonical (and not $\kappa$-Minkowski) phase space algebra and Special Relativity Lorentz transformation (and not $\kappa$-extended Lorentz transformation). The complete set of deformed symmetry generators are constructed that obeys an unmodified closed algebra but induce deformations in the symmetry transformations of the physical $\kappa$-Minkowski phase space variables. Furthermore, we demonstrate the usefulness and simplicity of this approach through a number of phenomenological applications both in classical and quantum mechanics. We also construct a Lagrangian for the $\kappa$-particle.
\end{abstract}




\section{Introduction:}

Evidence (see [1] for discussion and references) of ultra-high energy cosmic ray particles that violate the Greisen-Zatsepin-Kuzmin bound have compelled theorists to generalize the conventional energy-momentum dispersion law of particles,

$$
p^{2}=m^{2}
$$

based on principles of Special theory of Relativity (SR). The extension requires another observer independent dimensional parameter, apart from $c$, the velocity of light. The second parameter $(\kappa)$ is expected to be related to Planck energy. Based on this idea Amelino-Camelia [2] has pioneered an extended form of SR, popularly known as Deformed (or Doubly) Special theory of Relativity (DSR). It is very important to emphasize that the fundamental tenet of SR, i.e. equivalence of inertial frames of reference is kept intact in DSR whereas the Poincare algebra of SR is elevated to quantum (Hopf) $\kappa$-Poincare algebra in DSR. The effect of $\kappa$ appears in the explicit structures of Lorentz transformations (LT) in DSR, referred to here as $\kappa$-LT, which are non-linear for momenta and momenta-dependent for coordinates. Also DSR fully reduces to SR for energies much smaller that Planck energy, (or equivalently in the limit $\kappa \rightarrow \infty$ ).

DSR is intimately connected to another area of topical interest: Non-Commutative (NC) geometry [3, 4], where canonical Poisson Bracket structure of phase space variables is replaced by a more extended algebra. This is indeed satisfying because existence of a fundamental length scale ( $\sim$ Planck length) is needed to deal with quantum gravity ideas [5] and Black Hole physics [6] in a sensible way. This additional length scale can be implemented by an NC spacetime via generalized uncertainty relation [7. Exploiting the notion of duality in the context of Quantum Group ideas [8, 9, 10] or from a physical point particle Lagrangian perspective [11], it has been demonstrated that a DSR dispersion law is the Casimir invariant of a particular $\kappa$-deformed Poincare algebra and the latter is connected to an $\mathrm{NC}$ phase space in a unique way. It is important to note that in these examples the $\mathrm{NC}$ extension of the spacetime algebra is operatorial in nature,

$$
\left\{x_{\mu}, x_{\nu}\right\}=\theta_{\mu \nu}\left(x_{\alpha}, p_{\alpha}\right),
$$

( $p_{\alpha}$ being the momentum), in contrast to $\mathrm{NC}$ spaces of recent interest in High Energy Physics, where the extension is not operatorial [4],

$$
\left\{x_{\mu}, x_{\nu}\right\}=\theta_{\mu \nu}
$$

In the present paper we will dwell on the NC phase space aspect of DSR. Our approach [11] is more familiar to a physicist than the more mathematically oriented previous studies [8, 9, 10, We will focus on a particular form of NC spacetime, known as $\kappa$-Minkowski spacetime (and the associated phase space). In the sense of classical Poisson Brackets, the NC $\kappa$-Minkowski spacetime is defined as,

$$
\left\{x^{i}, x^{0}\right\}=\frac{x^{i}}{\kappa} ;\left\{x^{i}, x^{j}\right\}=0 .
$$

It should be mentioned that, even if one imposes the restrictions that Jacobi identities have to be maintained and that the structure should reduce to canonical algebra for $\kappa \rightarrow \infty$, the full $\kappa$-NC phase space algebra is not uniquely determined. There are distinct (and possibly inequivalent) 
representations that are connected by non-linear transformations [10]. The particular $\kappa$-NC phase space that we will use here was first studied in [12] and was further developed in [13] (in a restricted set up of $1+1$-dimensional toy model). In fact this phase space can be extracted from very general deformations considered by Lukierski et.al. (see the last reference in [8]). In [12, 13] the idea of a suitable definition for velocity of a particle, with a Magueijo-Smolin form [9] of modified dispersion relation,

$$
p^{2}=m^{2}\left[1-\frac{E}{\kappa}\right]^{2}
$$

$E$ being the particle energy, led to this NC phase space. Interestingly enough, this same phase space was embedded in a more general structure in an earlier work of one of us [11] where it was induced in a specific gauge that was used to fix the reparameterization invariance. (Similar framework has been used in various other contexts [14 to generate NC phase space.) We will follow the method used in [15] and obtain the expressions for finite $\kappa$-LTs. We will start from the infinitesimal $\kappa$-LTs induced by Lorentz generators in $\kappa$-space by explicit usage of the $\kappa$-NC algebra proposed here. The finite $\kappa$-LTs will be composed out of them by integration [15]. These specific $\kappa$-LTs were first derived in [16] from a different perspective. Also they were generated in [13] in the symplectic framework. We, on the other hand, will demonstrate that compatibility with the $\kappa$-NC phase space and associated $\kappa$-LTs necessarily lead to the generalized MS dispersion relation [9],

$$
p^{2}=m^{2}\left[1-\frac{(\eta p)}{\kappa}\right]^{2}=m^{2}\left[1-\frac{E}{\kappa}\right]^{2},
$$

now written in a covariant form with $\eta^{0}=1, \vec{\eta}=0$. We also show that the SR LT invariant interval has to be replaced by an expression that is DSR or $\kappa$-LT invariant [13].

In generalizations of Relativity theory in the form of introducing more than one observer independent scales, (e.g. in DSR there are two scales), generally the schemes are dominated by algebraic constructions based on the quantum (Hopf) $\kappa$-Poincare algebra [8, 10]. This is a deformation of the normal Poincare algebra of SR that is applicable in conventional phase space. 1 *-Product, an essential tool in the construction of quantum field theory in NC space, for the $\kappa$-NC spacetime has appeared in [18. This aspect of our model will appear elsewhere [19] and in the present work we will provide a thorough analysis of the deformed symmetry generators and their effects on the degrees of freedom.

Now comes the major part of our work where we introduce a mapping between $\left(x_{\mu}, p_{\mu}\right)$ the physical $\kappa$-Minkowski NC phase space and $\left(X_{\mu}, P_{\mu}\right)$ - a completely canonical phase space that obey normal Poisson Bracket algebra. We also show that $\left(X_{\mu}, P_{\mu}\right)$ obey SR LTs whereas $\left(x_{\mu}, p_{\mu}\right)$ are ruled by $\kappa$-LTs. Similar ideas have appeared before in $1+1$-dimensions in [13] and also in partially restricted ways in either coordinate space [20] or in momentum space [21]. However, the full canonical phase space, as has been constructed in the present work via the mapping, becomes a powerful tool. The idea of covariant phase space has also been applied [22] in some $\kappa$-extended field theory models to identify the conserved quantities.

\footnotetext{
${ }^{1}$ The construction of quantum field theories on a canonical NC space, (where $\theta_{\mu \nu}$ is a constant in $\left\{x_{\mu}, x_{\nu}\right\}=$ $\theta_{\mu \nu}$ ), has received a great impetus from the works of [17, that demonstrates the invariance of the theory under twisted Poincare algebra. This means that the representation content of $\mathrm{NC} x_{\mu}$ remains the same as there commutative counterpart. This assertion is very important from phenomenological model building point of view [17].
} 
On the one hand, existence of the associated canonical phase space is very intriguing and requires serious thinking, whereas on the other hand, it has a great practical utility in describing $\kappa$-LT kinematics and dynamics. It will also play an important role in quantization of the $\kappa$ particle. The idea is to write down $\mathrm{SR}$ or quantum relations in terms of $\left(X_{\mu}, P_{\mu}\right)$ and then use the inverse map to get corresponding relations in terms of $\left(x_{\mu}, p_{\mu}\right)$ variables, which (although $\mathrm{NC}$ ) are the relevant physical degrees of freedom. However, we also show that one has to be careful in extending SR kinematic relations to DSR relations since for interacting particles, the dynamic behavior becomes entwined with kinematics and isolated kinematic identities (as in SR case) are allowed only for free particle. Incidentally, even for the free $\kappa$-particle, the equation of motion (Newton's law) $\dot{p}_{\mu}=0$ is true but it is not at all obvious and we prove this from an action principle for the $\kappa$-particle. In the quantum case, we show from explicit examples that $\kappa$-effects are not observable in non-relativistic situations.

We provide a first order phase space Lagrangian for the $\kappa$-point particle and its geometric counterpart in coordinate space that has a higher derivative structure. (Higher derivative terms have also appeared in other DSR particle model [23].) Our model includes the two stand out features of DSR systems: NC spacetime and the modified dispersion relation, (taking $\kappa$ Minkowski spacetime and MS dispersion law as an example)2 2 . As we will show, here also the natural starting point is the normal relativistic point particle model in terms of $\left(X_{\mu}, P_{\mu}\right)$ which is rewritten in terms of $\mathrm{NC}$ coordinates $\left(x_{\mu}, p_{\mu}\right)$. We believe that the usage of this equivalent canonical framework, described extensively in the present work, to construct the deformed symmetry generators and the MS point particle Lagrangian, is new.

The paper is organized as follows: In Section II we introduce the $\kappa$-Minkowski phase space, the infinitesimal Lorentz generators and construct the finite $\kappa$-LTs for the phase space coordinates, i.e. $x_{\mu}$ and $p_{\mu}$. The $\kappa$-LT invariant MS dispersion law and coordinate interval are also identified. Section III is the main content of our work. In this section the canonical $\left(X_{\mu}, P_{\mu}\right)$ phase space is constructed out of $\left(x_{\mu}, p_{\mu}\right)$, the $\kappa$-NC phase space. The SR LT of the set $\left(X_{\mu}, P_{\mu}\right)$ are also shown explicitly. In Section IV we provide a thorough analysis of the set of deformed symmetry generators, comprising of generators of Translation, Rotation, Dilatation and Special Conformal Transformation, using the canonical approach. In Section V, we discuss kinematics in the $\kappa$ DSR context. Section VI is devoted to the construction of an action for the free $\kappa$ particle that is the starting point of all dynamical studies. The quantization problem is briefly touched upon in Section VII. The paper ends with a conclusion in Section VIII.

\section{II: Non-linear $\kappa$-Lorentz Transformations}

Let us start by introducing the $\kappa$-Minkowski phase space. We are in the classical framework and will interpret the phase space algebra as Poisson brackets. The general consensus is to refer a phase space as $\kappa$-Minkowski if it contains the following sector,

$$
\left\{x^{i}, x^{0}\right\}=\frac{x^{i}}{\kappa} ;\left\{x^{i}, x^{j}\right\}=0 ;\left\{x^{i}, p^{j}\right\}=-g^{i j} ;\left\{p^{\mu}, p^{\nu}\right\}=0 .
$$

Our metric is diag $g^{00}=-g^{i i}=1$ and $\kappa$ is the NC parameter. Rest of the phase space algebra

${ }^{2}$ The present model is a minimal construction in the sense that the previous model constructed by one of us [11] had a more elaborate phase space algebra. 
is given below,

$$
\left\{x^{0}, p^{i}\right\}=p^{i} / \kappa ;\left\{x^{i}, p^{0}\right\}=0 ;\left\{x^{0}, p^{0}\right\}=-1+p^{0} / \kappa .
$$

The above is rewritten in a covariant form,

$$
\begin{gathered}
\left\{x_{\mu}, x_{\nu}\right\}=\frac{1}{\kappa}\left(x_{\mu} \eta_{\nu}-x_{\nu} \eta_{\mu}\right), \\
\left\{x_{\mu}, p_{\nu}\right\}=-g_{\mu \nu}+\frac{1}{\kappa} \eta_{\mu} p_{\nu}, \quad\left\{p_{\mu}, p_{\nu}\right\}=0,
\end{gathered}
$$

where $\eta_{0}=1, \eta_{i}=0$. This algebra appeared in [12] and partially in [13]. Detailed studies of similar types of algebra are provided in [10. This algebra has emerged before in an earlier work of one of us [11] where it was embedded in a more general algebra. For $\kappa \rightarrow \infty$ one recovers the normal canonical phase space.

The angular momentum is defined in the normal way as,

$$
J_{\mu \nu}=x_{\mu} p_{\nu}-x_{\nu} p_{\mu}
$$

This is motivated by the fact that spatial sector of $\kappa$ - $\mathrm{NC}$ algebra in (7) remains unaffected. Furthermore, using (7) one can check that the Lorentz algebra is intact,

$$
\left\{J^{\mu \nu}, J^{\alpha \beta}\right\}=g^{\mu \beta} J^{\nu \alpha}+g^{\mu \alpha} J^{\beta \nu}+g^{\nu \beta} J^{\alpha \mu}+g^{\nu \alpha} J^{\mu \beta} .
$$

However, Lorentz transformations of $x_{\mu}$ and $p_{\mu}$ are indeed affected,

$$
\left\{J^{\mu \nu}, x^{\rho}\right\}=g^{\nu \rho} x^{\mu}-g^{\mu \rho} x^{\nu}+\frac{1}{\kappa}\left(p^{\mu} \eta^{\nu}-p^{\nu} \eta^{\mu}\right) x^{\rho} ;\left\{J^{\mu \nu}, p^{\rho}\right\}=g^{\nu \rho} p^{\mu}-g^{\mu \rho} p^{\nu}-\frac{1}{\kappa}\left(p^{\mu} \eta^{\nu}-p^{\nu} \eta^{\mu}\right) p^{\rho} .
$$

Notice that the extra terms appear only for $J^{0 i}$ and not for $J^{i j}$ so that only boost transformations are changed.

From now on we will use the $(x, y, x, t)$ notation (instead of the covariant one), which is more suitable for comparison with existing results. We define the infinitesimal transformation of a generic variable $O$ by,

$$
\delta O=\left\{\frac{1}{2} \omega_{\mu \nu} J^{\mu \nu}, O\right\},
$$

and only the parameter $\omega_{0 x}=\delta u$ is non-vanishing.

Let us start with the Lorentz transformation for energy-momentum vector $\left(E, p_{x}, p_{y}, p_{z}\right)$. The above considerations yield the following differential equations [15],

$$
\frac{d E}{d u}=-p_{x}+\frac{E p_{x}}{\kappa} ; \quad \frac{d p_{x}}{d u}=-E+\frac{p_{x}^{2}}{\kappa} ; \quad \frac{d p_{y}}{d u}=\frac{p_{y} p_{x}}{\kappa} ; \quad \frac{d p_{z}}{d u}=\frac{p_{z} p_{x}}{\kappa} .
$$

We rewrite the $E$ and $p_{x}$ equations as,

$$
\frac{d\left(E+p_{x}\right)}{d u}=-\left(E+p_{x}\right)+\frac{p_{x}}{\kappa}\left(E+p_{x}\right) ; \frac{d\left(E-p_{x}\right)}{d u}=\left(E-p_{x}\right)+\frac{p_{x}}{\kappa}\left(E-p_{x}\right),
$$

which reduces to,

$$
\frac{d}{d u}\left[\ln \left(\frac{\left(E+p_{x}\right)}{\left(E-p_{x}\right)}\right)\right]=-2 .
$$


Introducing the initial and final respectively as $E, p_{x}$ for $u=0$ and $E^{\prime}, p_{x}^{\prime}$, we obtain the following relation,

$$
E^{\prime}\left(1-A e^{-2 u}\right)=-p_{x}^{\prime}\left(1+A e^{-2 u}\right),
$$

where the constant $A$ is $A=\left(\frac{\left(E+p_{x}\right)}{\left(E-p_{x}\right)}\right)$. This allows us to deal with the $E$ (or $p_{x}$ ) equation separately and for $E$ we get,

$$
\frac{d E}{d u}=-p_{x}+\frac{E p_{x}}{\kappa}=\left(\frac{1-A e^{-2 u}}{1+A e^{-2 u}}\right)\left(E-\frac{E^{2}}{\kappa}\right) .
$$

The integration is trivial and we recover the finite Lorentz transformation for $E$ in $\kappa$-Minkowski spacetime:

$$
E^{\prime}=\frac{\gamma\left(E-v p_{x}\right)}{\alpha} ; \alpha=1+\frac{1}{\kappa}\left\{\left((\gamma-1) E-v \gamma p_{x}\right\}\right)
$$

In the above we define,

$$
\cosh (u)=\frac{1}{\sqrt{1-v^{2}}} \equiv \gamma ; \sinh (u)=v \gamma ; \quad e^{u}=\frac{1+v}{\sqrt{1-v^{2}}}=\gamma(1+v)
$$

where $v$ is the velocity of the primed frame with respect to the unprimed one. The relation (15) yields the $p_{x}$ transformation law,

$$
p_{x}^{\prime}=\frac{\gamma\left(p_{x}-v E\right)}{\alpha} .
$$

For the transformation law for the transverse momentum $p_{y}$ we start from

$$
\frac{d p_{y}^{\prime}}{d u}=\frac{p_{y}^{\prime} p_{x}^{\prime}}{\kappa}=-\frac{1}{\alpha} \frac{d \alpha}{d u} p_{y}^{\prime}
$$

where we have exploited the useful identity

$$
p_{x}^{\prime}=-\frac{\kappa}{\alpha} \frac{d \alpha}{d u}
$$

This is integrated to yield,

$$
p_{y}^{\prime}=\frac{p_{y}}{\alpha} .
$$

We collecting the momentum transformation laws together:

$$
E^{\prime}=\frac{\gamma\left(E-v p_{x}\right)}{\alpha} ; p_{x}^{\prime}=\frac{\gamma\left(p_{x}-v E\right)}{\alpha} ; p_{y}^{\prime}=\frac{p_{y}}{\alpha} ; p_{z}^{\prime}=\frac{p_{z}}{\alpha} .
$$

These transformations have been derived before in [16] from different considerations and we have based our analysis completely on the NC $\kappa$-Minkowski phase space. Our method was used in similar context by [15] but with a different representation of $\kappa$-Minkowski phase space algebra.

Before proceeding to derive the $\kappa$-LT for the coordinates $x_{\mu}$, let us first find out the new dispersion law that is $\kappa$-LT invariant. Scanning the following infinitesimal transformation rules,

$$
\left\{\frac{1}{2} J_{\mu \nu}, p^{2}\right\}=\frac{p^{2}}{\kappa}\left(\eta_{\mu} p_{\nu}-\eta_{\nu} p_{\mu}\right) ; \quad\left\{\frac{1}{2} J_{\mu \nu},(\eta p)\right\}=-\left(1-\frac{(\eta p)}{\kappa}\right)\left(\eta_{\mu} p_{\nu}-\eta_{\nu} p_{\mu}\right)
$$


we find the following combination to be invariant:

$$
\left\{\frac{1}{2} J_{\mu \nu}, \frac{p^{2}}{\left(1-\frac{(\eta p)}{\kappa}\right)^{2}}\right\}=0 .
$$

The finite $\kappa$-LTs also yields

$$
\left(p^{2}-m^{2}\left(1-\frac{(\eta p)}{\kappa}\right)^{2}\right)^{\prime}=\frac{1}{\alpha^{2}}\left(p^{2}-m^{2}\left(1-\frac{(\eta p)}{\kappa}\right)^{2}\right),
$$

confirming that the new $\kappa$-LT invariant dispersion law is

$$
p^{2}=m^{2}\left(1-\frac{(\eta p)}{\kappa}\right)^{2}
$$

This is the MS dispersion law [9] mentioned at the beginning (44). We recover all the conventional relations in the $\kappa \rightarrow \infty$ limit.

Now we discuss the generalized spacetime transformation relations. We follow the same procedure as before and starting from (10) obtain the differential equations for $t$ and $x$,

$$
\frac{d t^{\prime}}{d v}=-x^{\prime}-\frac{t^{\prime} p_{x}^{\prime}}{\kappa}=-x^{\prime}+\frac{t^{\prime}}{\alpha} \frac{d \alpha}{d v} ; \quad \frac{d x^{\prime}}{d v}=-t^{\prime}-\frac{x^{\prime} p_{x}^{\prime}}{\kappa}=-t^{\prime}+\frac{x^{\prime}}{\alpha} \frac{d \alpha}{d v},
$$

which are reexpressed as

$$
\frac{d}{d v}\left(\frac{t^{\prime}}{\alpha}\right)=-\frac{x^{\prime}}{\alpha} ; \quad \frac{d}{d v}\left(\frac{x^{\prime}}{\alpha}\right)=-\frac{t^{\prime}}{\alpha} .
$$

A further differentiation separates the variables and we get,

$$
\frac{d^{2}}{d v^{2}}\left(\frac{x^{\prime}}{\alpha}\right)=\frac{x^{\prime}}{\alpha} ; \quad \frac{d^{2}}{d v^{2}}\left(\frac{t^{\prime}}{\alpha}\right)=\frac{t^{\prime}}{\alpha}
$$

giving rise to the solutions,

$$
x^{\prime}=\alpha \gamma(x-v t), \quad t^{\prime}=\alpha \gamma(t-v x)
$$

where the initial and final conditions respectively are $x, t$ for $v=0$ and $x^{\prime}, t^{\prime}$. For the transverse coordinate $y$, the differential equation,

$$
\frac{d y^{\prime}}{d v}=-\frac{y^{\prime} p_{x}^{\prime}}{\kappa}=\frac{y^{\prime}}{\alpha} \frac{d \alpha}{d v}
$$

induces the transformation,

$$
y^{\prime}=\alpha y .
$$

Hence the $\kappa$-LTs for spacetime coordinates appear as,

$$
t^{\prime}=\alpha \gamma(t-v x) ; \quad x^{\prime}=\alpha \gamma(x-v t), \quad y^{\prime}=\alpha y, \quad z^{\prime}=\alpha z .
$$

These $\kappa$-LTs are precisely the ones derived in [16] in a different way where imposing the invariance of $x^{\mu} p_{\mu}$ was the starting point and partially derived in [13] in a Hamiltonian sysplectic 
framework. In the present paper this derivation is more systematic and rests solely on the new form of $\kappa$-algebra (7) introduced here.

As in the dispersion relation, once again we look for an invariant quantity that will generalize the conventional distance and we find that under the $\kappa$-LT (32),

$$
\left(x^{2}\left(1-\frac{(\eta p)}{\kappa}\right)^{2}\right)^{\prime}=x^{2}\left(1-\frac{(\eta p)}{\kappa}\right)^{2} .
$$

Hence the invariant length $|S|$ is generalized to

$$
s^{2}=x^{2}\left(1-\frac{(\eta p)}{\kappa}\right)^{2}
$$

This is one of the important results of the present paper. Its $1+1$-dimensional analogue was suggested in [13].

\section{III: Canonical Variables}

In this section we will introduce a new set of phase space variables which obey canonical Poisson brackets are transform in the conventional way under SR Lorentz transformation. Somewhat similar considerations in parts have appeared before in [13, 20, 21] but exhaustive study of the full canonical phase space as presented here is new. Indeed, these variables are composites of phase space coordinates will have to suitable ordered upon quantization. But, in the classical framework they will prove to be very convenient and they drastically simplify the computations while analyzing phenomenological consequences of the modified Lorentz transformations. We will return to the quantum case at the end.

The two invariant quantities that we derived in (44.34) suggest the forms of these canonical avatars:

$$
X_{\mu} \equiv x_{\mu}\left(1-\frac{(\eta p)}{\kappa}\right)=x_{\mu}\left(1-\frac{E}{\kappa}\right) ; \quad P_{\mu} \equiv \frac{p_{\mu}}{\left(1-\frac{(\eta p)}{\kappa}\right)}=\frac{p_{\mu}}{\left(1-\frac{E}{\kappa}\right)} .
$$

We remind that the variables on the right hand side obey $\kappa$-LT laws. Using the NC algebra (7) it is easy to check the following:

$$
\left\{X_{\mu}, P_{\nu}\right\}=-g_{\mu \nu} ; \quad\left\{X_{\mu}, X_{\nu}\right\}=\left\{P_{\mu}, P_{\nu}\right\}=0
$$

Hence the $X, P$ phase space is canonical. The above relations in (35) are invertible,

$$
x_{\mu}=X_{\mu}\left(1+\frac{(\eta P)}{\kappa}\right)=X_{\mu}\left(1+\frac{P_{0}}{\kappa}\right) ; \quad p_{\mu}=\frac{P_{\mu}}{\left(1+\frac{(\eta P)}{\kappa}\right)}=\frac{P_{\mu}}{\left(1+\frac{P_{0}}{\kappa}\right)} .
$$

Next we consider Lorentz transformations of the canonical variables and find, for example,

$$
\begin{aligned}
T^{\prime} & =t^{\prime}\left(1-\frac{E}{\kappa}\right)^{\prime}=\gamma \bar{\alpha}(t-v x)\left[1-\frac{\gamma}{\kappa \bar{\alpha}}\left(E-v p_{x}\right)\right] \\
& =\gamma\left[t\left(1-\frac{E}{\kappa}\right)-v x\left(1-\frac{E}{\kappa}\right)\right]=\gamma(T-v X),
\end{aligned}
$$


where $\bar{\alpha}=\alpha(-v)$ and we have used the identity $(\bar{\alpha})^{-1}=\alpha^{\prime}$. In an identical fashion we can show,

$$
X^{\prime}=x^{\prime}\left(1-\frac{E}{\kappa}\right)^{\prime}=\gamma(X-v T) ; \quad Y^{\prime}=y^{\prime}\left(1-\frac{E}{\kappa}\right)^{\prime}=Y ; \quad Z^{\prime}=Z .
$$

Exploiting similar considerations we also ascertain that,

$$
\begin{gathered}
P_{0}^{\prime}=\frac{E^{\prime}}{\left(1-\frac{E}{\kappa}\right)^{\prime}}=\gamma\left(P_{0}-v P_{x}\right) ; \quad P_{x}^{\prime}=\frac{p_{x}^{\prime}}{\left(1-\frac{E}{\kappa}\right)^{\prime}}=\gamma\left(P_{x}-v P_{0}\right) ; \\
P_{y}^{\prime}=\frac{p_{y}^{\prime}}{\left(1-\frac{E}{\kappa}\right)^{\prime}}=P_{y}, \quad P_{z}^{\prime}=P_{z} .
\end{gathered}
$$

To further convince ourselves about the validity of the canonical variable approach let us study the group property of these new Lorentz transformations. Consider, for example, two successive $\kappa$-LT' on $x$,

$$
\begin{gathered}
x=\alpha_{1}^{\prime}\left(C_{1} x^{\prime}+S_{1} t^{\prime}\right) ; \quad t=\alpha_{1}^{\prime}\left(C_{1} t^{\prime}+S_{1} x^{\prime}\right), \\
x^{\prime}=\alpha_{2}^{\prime \prime}\left(C_{2} x^{\prime \prime}+S_{2} t^{\prime \prime}\right) ; \quad t^{\prime}=\alpha_{2}^{\prime \prime}\left(C_{2} t^{\prime \prime}+S_{2} x^{\prime \prime}\right),
\end{gathered}
$$

where

$$
C_{1}=\cosh \left(u_{1}\right)=\frac{1}{\sqrt{1-v_{1}^{2}}}=\gamma_{1} ; S_{1}=\sinh \left(u_{1}\right)=v_{1} \gamma_{1} ; \alpha_{1}^{\prime}=1-\frac{1}{\kappa}\left\{\left(1-C_{1}\right) E^{\prime}-S_{1} p_{x}^{\prime}\right\}
$$

and so on. We will also require the energy-momentum $\kappa$-LTs,

$$
E^{\prime}=\frac{1}{\alpha_{2}^{\prime \prime}}\left(C_{2} E^{\prime \prime}+S_{2} p_{x}^{\prime \prime}\right) ; \quad p_{x}^{\prime}=\frac{1}{\alpha_{2}^{\prime \prime}}\left(C_{2} p_{x}^{\prime \prime}+S_{2} E^{\prime \prime}\right)
$$

Concentrating on the $x$-transformation, the above will lead to,

$$
x=\alpha_{1}^{\prime}\left[C_{1}\left\{\alpha_{2}^{\prime \prime}\left(C_{2} x^{\prime \prime}+S_{2} t^{\prime \prime}\right)\right\}+S_{1}\left\{\alpha_{2}^{\prime \prime}\left(C_{2} t^{\prime \prime}+S_{2} x^{\prime \prime}\right)\right\}\right]=\alpha_{(1+2)}^{\prime \prime}\left[C_{(1+2)} x^{\prime \prime}+S_{(1+2)} t^{\prime \prime}\right],
$$

where $(1+2)$ stands for $\left(v_{1}+v_{2}\right)$. However, in terms of the canonical variables we should simply obtain,

$$
X=C_{(1+2)} X^{\prime \prime}+S_{(1+2)} T^{\prime \prime}
$$

which, when expressed in terms of $\mathrm{NC}$ physical variables yields,

$$
\begin{gathered}
\left(1-\frac{E}{\kappa}\right) x=C_{(1+2)}\left(1-\frac{E^{\prime \prime}}{\kappa}\right) x^{\prime \prime}+S_{(1+2)}\left(1-\frac{E^{\prime \prime}}{\kappa}\right) t^{\prime \prime} \\
\Rightarrow x=\frac{\left(1-\frac{E^{\prime \prime}}{\kappa}\right)}{\left(1-\frac{E}{\kappa}\right)} C_{(1+2)} x^{\prime \prime}+S_{(1+2)} t^{\prime \prime}=\alpha_{(1+2)}^{\prime \prime}\left(C_{(1+2)} x^{\prime \prime}+S_{(1+2)} t^{\prime \prime}\right) .
\end{gathered}
$$

This is same as (43). Hence the group property is established for $\kappa$-LTs and also the fact that the canonical $X_{\mu}, P_{\mu}$ variables obey SR LTs.

The important lesson that we learn from the above discussion is that the $\left(X_{\mu}, P_{\mu}\right)$ phase space is truly canonical in the sense that it satisfies canonical Poison brackets and moreover obeys normal $(\kappa \rightarrow \infty)$ SR LTs. Hence, as far as classical physics is concerned, we can directly borrow the SR kinematical laws (by writing them in terms of $\left(X_{\mu}, P_{\mu}\right)$, and subsequently deduce 
the laws in $\kappa$-NC spacetime by rewriting them in terms of $\left(x_{\mu}, p_{\mu}\right)$, using the mapping (35). We will put this idea in immediate use in the next section where we obtain the deformed symmetry generators. However, as we will discuss in later sections, one has to be more careful while establishing dynamical laws, even though the basic approach remains the same. In the last section, we will briefly comment on the quantization problem, which is very interesting.

\section{IV: Deformed Symmetry Generators}

Let us understand the fate of the conventional symmetry principles in the $\kappa$-extended particle model. We will see that we need deformed symmetry generators.

In the conventional case, the phase space algebraic structure of the point particle is invariant under the following symmetry transformations: translation, Lorentz rotation, dilation and special conformal transformation. On the other hand, the particle dispersion relation $P^{2}-$ $m^{2}=0$ enjoys invariance under translation and Lorentz rotation, and the mass term $m$ breaks the symmetry under dilation and special conformal transformation. Finally, the symmetry generators satisfy a closed algebra among themselves.

In the $\kappa$-particle model our aim is to construct the generators in the $\kappa$ - $\mathrm{NC}$ space that preserve invariances of both the $\kappa$-NC phase space algebra (17) and the structure of the algebra among generators (see below in (46)) ). Then we will check how the $\kappa$-modified dispersion relation (MS relation (41) in the present case) is affected. Once again the canonical $\left(X_{\mu}, P_{\mu}\right)$ variables will do the trick. The idea is to first write down the generators in terms of $\left(X_{\mu}, P_{\mu}\right)$ degrees of freedom using the conventional form of the generators, (i.e. that of normal particle in normal phase space). They will obviously satisfy the standard closed algebra among generators:

$$
\begin{gathered}
\left\{J^{\mu \nu}, J^{\alpha \beta}\right\}=g^{\mu \beta} J^{\nu \alpha}+g^{\mu \alpha} J^{\beta \nu}+g^{\nu \beta} J^{\alpha \mu}+g^{\nu \alpha} J^{\mu \beta} ;\left\{J^{\mu \nu}, T^{\sigma}\right\}=g^{\nu \sigma} T^{\mu}-g^{\mu \sigma} T^{\nu} ; \\
\left\{J^{\mu \nu}, D\right\}=0 ;\left\{J^{\mu \nu}, K^{\sigma}\right\}=2 D\left(g^{\nu \sigma} X^{\mu}-g^{\mu \sigma} X^{\nu}\right)-X^{2}\left(g^{\nu \sigma} T^{\mu}-g^{\mu \sigma} T^{\nu}\right) ; \\
\left\{T^{\mu}, T^{\nu}\right\}=0 ;\left\{T^{\mu}, D\right\}=T^{\mu} ;\left\{T^{\mu}, K^{\nu}\right\}=2 D g^{\mu \nu}-2 J^{\mu \nu} ; \\
\{D, D\}=0 ;\left\{D, K^{\mu}\right\}=K^{\mu} ;\left\{K^{\mu}, K^{\nu}\right\}=0,
\end{gathered}
$$

where $J_{\mu \nu}, T_{\mu}, D$ and $K_{\mu}$ stand for generators of Lorentz rotation, translation, dilation and special conformal transformation respectively. Their structures are given by,

$$
\begin{gathered}
J_{\mu \nu}=X_{\mu} P_{\nu}-X_{\nu} P_{\mu} ; T_{\mu}=P_{\mu} ; \quad D=(X P) ; \\
K_{\mu}=2(X P) X_{\mu}-X^{2} P_{\mu} .
\end{gathered}
$$

Next we exploit the map $\left(X_{\mu}, P_{\mu}\right) \rightarrow\left(x_{\mu}, p_{\mu}\right)$ given in (35) to rewrite the generators in the $\kappa$-NC spacetime:

$$
\begin{gathered}
j_{\mu \nu}=x_{\mu} p_{\nu}-x_{\nu} p_{\mu} ; \quad t_{\mu}=\frac{p_{\mu}}{1-(\eta p) / \kappa} ; \quad ; \quad d=(x p) ; \\
k_{\mu}=(1-(\eta p) / \kappa)\left[2(x p) x_{\mu}-x^{2} p_{\mu}\right] .
\end{gathered}
$$

By construction, the generators in (48) will satisfy the same algebra (46) provided one uses the $\kappa-N C$ algebra (7). These are the deformed generators. The infinitesimal transformation operators are,

$$
j=\frac{1}{2} a^{\mu \nu} j_{\mu \nu}=\frac{1}{2} a^{\mu \nu}\left(x_{\mu} p_{\nu}-x_{\nu} p_{\mu}\right) ; t=a^{\mu} t_{\mu}=\frac{(a p)}{1-(\eta p) / \kappa} ; d=a(x p) ;
$$




$$
k=a^{\mu} k_{\mu}=(1-(\eta p) / \kappa)\left[2(x p)(a x)-x^{2}(a p)\right],
$$

where generically $a$ denotes the infinitesimal parameter. Using the definition of small change in $A$ due to transformation $\delta_{b}$ as,

$$
\delta_{b} A=\left\{\delta_{b}, A\right\}
$$

we compute the explicit forms of transformations:

$$
\begin{gathered}
\delta_{j} x_{\mu}=a^{\alpha \beta}\left(g_{\beta \mu} x_{\alpha}+\frac{1}{\kappa} p_{\alpha} \eta_{\beta} x_{\mu}\right) ; \delta_{j} p_{\mu}=a^{\alpha \beta}\left(g_{\beta \mu} p_{\alpha}-\frac{1}{\kappa} p_{\alpha} \eta_{\beta} p_{\mu}\right), \\
\delta_{t} x_{\mu}=\frac{a_{\mu}}{1-(\eta p) / \kappa} ; \delta_{t} p_{\mu}=0 \\
\delta_{d} x_{\mu}=a(1-(\eta p) / \kappa) x_{\mu} ; \delta_{d} p_{\mu}=-a(1-(\eta p) / \kappa) p_{\mu}, \\
\delta_{k} x_{\mu}=(1-(\eta p) / \kappa)\left[2(a x) x_{\mu}-x^{2} a_{\mu}+\frac{2}{\kappa} x_{\mu}(-(x p)(a \eta)-(\eta p)(a x)+(\eta x)(a p))\right] \\
\delta_{k} p_{\mu}=2(1-(\eta p) / \kappa)\left[\left((a p) x_{\mu}-(a x) p_{\mu}-(x p) a_{\mu}\right)+\frac{1}{\kappa} p_{\mu}((x p)(a \eta)+(\eta p)(a x)-(\eta x)(a p))\right] .
\end{gathered}
$$

Clearly the variations differ from their commutative spacetime counterpart. Next we want to ascertain that the $\kappa$-NC algebra (17) is stable under the above symmetry operations. This is done by checking the validity of the identity,

$$
\{A, B\}=C \Rightarrow \delta_{b}\{A, B\}=\delta_{b} C,
$$

or more explicitly,

$$
\left\{\delta_{b} A, B\right\}+\left\{A, \delta_{b} B\right\}=\delta_{b} C .
$$

In the above we refer to (7) for $\{A, B\}=C$ and (51,54) for $\delta_{b}$. A straightforward but tedious calculation shows that the above identity is, indeed, valid. This assures us about the consistency of the whole procedure.

Regarding the behavior of the MS dispersion law (44) we have already checked that it is $\kappa$-Lorentz invariant. It is also trivially translation invariant. The variation under dilatation is given by,

$$
\delta_{d}\left(p^{2}-m^{2}(1-(\eta p) / \kappa)^{2}\right)=-2(1-(\eta p) / \kappa)\left[p^{2}+m^{2}(1-(\eta p) / \kappa) \frac{(\eta p)}{\kappa}\right]=-2 p^{2},
$$

where the MS relation is imposed on the right hand side. In fact this variation is structurally identical to that of the normal particle showing that in $\kappa$-NC spacetime also the mass terms breaks dilation invariance in the same way.

For special conformal transformation, the variation is given by,

$$
\begin{aligned}
\delta_{k}\left(p^{2}-m^{2}(1-(\eta p) / \kappa)^{2}\right)=2(1 & -(\eta p) / \kappa)\left[-(a x) p^{2}+\frac{1}{\kappa}\left(p^{2}-m^{2}(1-(\eta p) / \kappa)^{2}\right)((x p)(a \eta)\right. \\
& +(\eta p)(a x)-(\eta x)(a p))] .
\end{aligned}
$$

If we impose the MS law on the right hand side of (58) we find the variation to be,

$$
\delta_{k}\left(p^{2}-m^{2}(1-(\eta p) / \kappa)^{2}\right)=-2(1-(\eta p) / \kappa)(a x) p^{2},
$$


which very clearly mimics the normal particle characteristics.

The above results indicate how the $\kappa$-extension modifies the mathematical structure of the normal (Special Theory) relativistic particle. At the same time we have explicitly constructed the full set of deformed symmetry generators in $\kappa$-spacetime that have all the attributes of these generators in normal comutative spacetime.

\section{V: $\kappa$-Lorentz Transformation Phenomenology}

Let us start by discussing an important issue: the $\kappa$-LT of velocity, that will lead to the velocity addition theorem. In our canonical setup, the three velocity (in the primed frame) can be defined in the conventional way,

$$
\vec{U}^{\prime}=U_{X}^{\prime} \hat{i}+U_{Y}^{\prime} \hat{j}+U_{Z}^{\prime} \hat{k} ; \quad U_{X}^{\prime}=\frac{d X^{\prime}}{d T^{\prime}}, \ldots
$$

Hence, in terms of these variables, the velocity (in an unprimed frame, moving with velocity $v$ in $X$-direction) will obey conventional Special Theory relations,

$$
\begin{gathered}
\vec{W}=W_{X} \hat{i}+W_{Y} \hat{j}+W_{Z} \hat{k} ; \quad W_{X}=\frac{d X}{d T}, . . \\
W_{X}=\frac{U_{X}^{\prime}+v}{1+U_{X}^{\prime} v} ; \quad W_{X}=\frac{U_{X}^{\prime}+v}{1+U_{X}^{\prime} v} ; \quad W_{Y}=\frac{U_{Y}^{\prime}}{1+U_{X}^{\prime} v} ; \quad W_{Z}=\frac{U_{Z}^{\prime}}{1+U_{X}^{\prime} v} .
\end{gathered}
$$

Let us now map this relation to $\mathrm{NC}\left(x_{\mu}, p_{\mu}\right)$ space. From the mapping (35) it is clear that the velocity components are unchanged,

$$
\frac{d X}{d T}=\frac{d x}{d t}, \ldots \frac{d X^{\prime}}{d T^{\prime}}=\frac{d x^{\prime}}{d t^{\prime}}, \ldots
$$

which means that the velocity transformation laws will not change in the $\kappa$-spacetime and in particular the velocity addition law will remain unchanged.

However, in reality the analysis is more tricky and so far as we have discussed, it validity is restricted only to free $\kappa$-particle. In fact this statement also needs to be proved which we do in Section IV by considering a specific model for a $\kappa$-particle. To understand the problem let us follow the derivation of the velocity addition rule more closely. From $\kappa$-LT the velocity components are related by,

$$
\begin{gathered}
x=\gamma \alpha^{\prime}\left(x^{\prime}+v t^{\prime}\right) \\
\Rightarrow \frac{d x}{d t}=\gamma \alpha^{\prime} \frac{d t^{\prime}}{d t}\left(\frac{d x^{\prime}}{d t^{\prime}}+v\right)+\psi \Rightarrow w_{x}=\gamma \alpha^{\prime} \frac{d t^{\prime}}{d t}\left(u_{x}^{\prime}+v\right)+\psi .
\end{gathered}
$$

The piece $\psi$ can come from $(d E) /(d t)$ if the particle is interacting. Note that the $(d E) /(d t)$ term will contribute to $O(1 / \kappa)$. For free particles we will show in Section V that $(d E) /(d t)=0$ and so it will not affect (63). Hence, for free particle we have,

$$
w_{x}=\gamma \alpha^{\prime} \frac{d t^{\prime}}{d t}\left(u_{x}^{\prime}+v\right) .
$$

In a similar way, for the free particle we will have,

$$
\frac{d t^{\prime}}{d t}=\frac{\gamma}{\alpha^{\prime}}\left(1-v w_{x}\right)
$$


where we have taken $p_{x}$ to be time independent, which we prove later. Substituting (65) in (64) we get

$$
w_{x}=\frac{u_{x}^{\prime}+v}{1+u_{x}^{\prime} v}
$$

Similar results will follow for other velocity components.

In case of $\kappa$-LT for acceleration components $\left(a_{x}\right)$ for the free particle we find

$$
a_{x}=\frac{1}{\alpha^{\prime}} \frac{a_{x}^{\prime}}{\left(\gamma^{2}\left(1+u_{x}^{\prime} v\right)\right)^{3}} .
$$

Hence the $\kappa$-LT law for acceleration is changed even for the free particle, in contrast to the velocity relation, which remains unaltered. The $\kappa$-modification for the other components of accleration are same in nature.

From the structure of $\kappa$-LT invariants (25,33) and the mappings (35), it is clear that the phase space variables $x_{\mu}$ and $p_{\mu}$ get mixed up in an inseparable way. This is the reason why kinematic laws are not isolated from the dynamical behavior of the particle. In case the particle is not free, the simplest possibility is that it is under the influence of an external force $\vec{F}$. In that case we should use

$$
\frac{d E}{d t}=\vec{F} \cdot \vec{w}
$$

and so there will $O\left(\frac{1}{\kappa}\right)$ corrections, depending on the force, in all the relations.

We can also study Lorentz contraction. We find that

$$
X_{2}-X_{1}=\gamma\left(X_{2}^{\prime}-X_{1}^{\prime}\right) \Rightarrow x_{2}-x_{1}=\gamma\left(\alpha_{2}^{\prime} x_{2}^{\prime}-\alpha_{1}^{\prime} x_{1}^{\prime}\right)
$$

where 2 and 1 refer to two spacetime positions and $\alpha_{2}$ and $\alpha_{1}$ are the values of $\alpha$ at positions 2 and 1 respectively. Once again for a free particle we use,

$$
E_{2}=E_{1} ; \quad\left(p_{x}\right)_{2}=\left(p_{x}\right)_{1},
$$

and find the modified Lorentz contraction law for length $l$ as,

$$
l^{\prime}=\frac{l}{\gamma}\left[1-\frac{1}{\kappa}\left\{(1-\gamma) E+v \gamma p_{x}\right\}\right]
$$

applicable to free particle.

It is curious to see that in the normal $(\kappa \rightarrow \infty)$ case,

$$
l^{\prime}=\frac{l}{\gamma} \approx l\left(1-\frac{v^{2}}{2}\right)+O\left(v^{3}\right),
$$

and in the $\kappa$-spacetime, keeping terms up to $O(v)$ in the non-relativistic limit, we have,

$$
l^{\prime} \approx l\left(1-\frac{v p_{x}}{\kappa}\right) \sim l\left(1-\frac{m v^{2}}{\kappa}\right)
$$

for $p_{x} \sim m v$.

In the next section we will formulate an explicit model of a point particle that has a $\kappa$ Minkowski NC phase space (7) and Magueijo-Smolin dispersion law (25). This will help us to 
understand how, some of the assumptions that we have made in this section, regarding the particles energy and momentum, are concretely realized.

\section{VI: Lagrangian for $\kappa$-Particle}

In this section we construct a Lagrangian for the $\kappa$-particle. This has been a topic of recent interest and several authors [14, 11] have proposed models for particles with NC phase space of different structures. However, the model we propose here for $\kappa$-NC phase space is quite elegant and can be expressed in a closed form.

Again the canonical variable approach becomes convenient since we are sure that the relativistic free particle action in terms of canonical $\left(X_{\mu}, P_{\mu}\right)$ degrees of freedom will be,

$$
L=\left(P^{\mu} \dot{X}_{\mu}\right)-\lambda\left(P^{2}-m^{2}\right) .
$$

We now convert this $L$ to a function depending on physical $\kappa$-NC phase space coordinates:

$$
\begin{aligned}
L & =\left(\frac{p^{\mu}}{1-\frac{(\eta p)}{\kappa}}\right)\left(x_{\mu}\left(1-\frac{\eta p)}{\kappa}\right)\right) \cdot-\frac{\lambda}{2}\left(\frac{p^{2}}{\left(1-\frac{(\eta p)}{\kappa}\right)^{2}}-m^{2}\right) \\
& =(p \dot{x})-\frac{(p x)(\eta \dot{p})}{\kappa\left(1-\frac{(\eta p)}{\kappa}\right)}-\frac{\lambda}{2}\left(p^{2}-m^{2}\left(1-\frac{(\eta p)}{\kappa}\right)^{2}\right),
\end{aligned}
$$

where we have redefined the arbitrary multiplier $\lambda$. Our claim is that the symplectic structure in (73) will induce the $\kappa$-NC phase space algebra and the $\lambda$-term will obviously impose the MS mass shell condition. A slightly differenct form of symplectic structure in $1+1$-dimensions is given in [13. We now proceed to demonstrate the former in Dirac's Hamiltonian constraint analysis scheme [24].

In the above first order Lagrangian (73) $x_{\mu}$ and $p_{\mu}$ are treated as independent variables. The conjugate momenta are,

$$
\pi_{\mu}^{x}=\frac{\partial L}{\partial \dot{x}^{\mu}}=p_{\mu} ; \quad \pi_{\mu}^{p}=\frac{\partial L}{\partial \dot{p}^{\mu}}=-\frac{(p x)}{\kappa\left(1-\frac{(\eta p)}{\kappa}\right)} \eta_{\mu} .
$$

The only nontrivial Poisson brackets are,

$$
\left\{x_{\mu}, \pi_{\nu}^{x}\right\}=\left\{p_{\mu}, \pi_{\nu}^{p}\right\}=-g_{\mu \nu} .
$$

The momenta equation (74) shows that there are two sets of constraints:

$$
\psi_{\mu}^{1} \equiv \pi_{\mu}^{x}-p_{\mu} \approx 0 ; \quad \psi_{\mu}^{2} \equiv \pi_{\mu}^{p}+\frac{(p x)}{\kappa\left(1-\frac{(\eta p)}{\kappa}\right)} \eta_{\mu} \approx 0 .
$$

In the terminology of Dirac constraint analysis [24], the non-commutating constraints are termed as Second Class Constraints (SCC) and the commutating constraints, that induce local gauge invariance, are First Class Constraints (FCC). In the presence of SCCs $\left(\psi^{1}, \psi^{2}\right)$ that do not commute (as Poisson Brackets), $\left\{\psi^{1}, \psi^{2}\right\} \neq 0$, the modified symplectic structure (or Dirac Brackets) are defined in the following way,

$$
\{A, B\}^{*}=\{A, B\}-\left\{A, \psi^{i}\right\}\left\{\psi^{i}, \psi^{j}\right\}^{-1}\left\{\psi^{j}, B\right\},
$$


where $\left\{\psi^{i}, \psi^{j}\right\}$ refers to the constraint matrix.

For the set of constraints (76) the constraint matrix is

$$
\left\{\psi_{\mu}^{i}, \psi_{\nu}^{j}\right\}=\left(\begin{array}{cc}
0 & g_{\mu \nu}+\frac{p_{\mu} \eta_{\nu}}{\kappa\left(1-\frac{(\eta p)}{\kappa}\right)} \\
-g_{\mu \nu}-\frac{p_{\nu} \eta_{\mu}}{\kappa\left(1-\frac{(\eta p)}{\kappa}\right)} & \frac{x_{\mu} \eta_{\nu}-x_{\nu} \eta_{\mu}}{\kappa\left(1-\frac{(\eta p)}{\kappa}\right)}
\end{array}\right) .
$$

The inverse matrix is computed to be,

$$
\left\{\psi_{\nu}^{i}, \psi_{\lambda}^{j}\right\}^{-1}=\left(\begin{array}{cc}
\frac{1}{\kappa}\left(x_{\nu} \eta_{\lambda}-x_{\lambda} \eta_{\nu}\right) & -g_{\nu \lambda}+\frac{1}{\kappa} \eta_{\nu} p_{\lambda} \\
g_{\nu \lambda}-\frac{1}{\kappa} \eta_{\lambda} p_{\nu} & 0
\end{array}\right) .
$$

Exploiting the definition (77) we obtain the Dirac Brackets,

$$
\left\{x_{\mu}, x_{\nu}\right\}=\frac{1}{\kappa}\left(x_{\mu} \eta_{\nu}-x_{\nu} \eta_{\mu}\right) ;\left\{x_{\mu}, p_{\nu}\right\}=-g_{\mu \nu}+\frac{1}{\kappa} \eta_{\mu} p_{\nu} ; \quad\left\{p_{\mu}, p_{\nu}\right\}=0,
$$

which is nothing but the $\kappa$-NC phase space introduced at the beginning (77). Hence $L$ in (73) correctly reproduces the $\kappa$-NC symplectic structure.

It is also very useful to have the Nambu-Goto form of action comprising only of coordinate space variables for the $\kappa$-particle. This can be recovered by eliminating $p_{\mu}$ and $\lambda$ from $L$ given in (73), which we now proceed to do.

The variational equations of motion from (73) are,

$$
\begin{gathered}
\dot{x}_{\mu}-\frac{(\eta \dot{p})}{\kappa\left(1-\frac{(\eta p)}{\kappa}\right)} x_{\mu}+\frac{(p x) \cdot}{\kappa\left(1-\frac{(\eta p)}{\kappa}\right)} \eta_{\mu}-\lambda\left(p_{\mu}+\frac{m^{2}}{\kappa}\left(1-\frac{(\eta p)}{\kappa}\right) \eta_{\mu}\right)=0, \\
\dot{p}_{\mu}+\frac{(\eta \dot{p})}{\kappa\left(1-\frac{(\eta p)}{\kappa}\right)} p_{\mu}=0 .
\end{gathered}
$$

Rewriting (82) in the form,

$$
\dot{p}_{\sigma}\left(g^{\sigma \mu}+\frac{\eta^{\sigma p^{\mu}}}{\kappa\left(1-\frac{(\eta p)}{\kappa}\right)}\right) \equiv \dot{p}_{\sigma} G^{\sigma \mu}=0,
$$

we observe that $G^{-1}$ exists and hence (82) yields,

$$
\dot{p}_{\mu}=0
$$

This condition considerably simplifies (81) to,

$$
\dot{x}_{\mu}+\frac{(p \dot{x})}{\kappa\left(1-\frac{(\eta p)}{\kappa}\right)} \eta_{\mu}=\lambda\left(p_{\mu}+\frac{m^{2}}{\kappa}\left(1-\frac{(\eta p)}{\kappa}\right) \eta_{\mu}\right) \Rightarrow\left(1-\frac{(\eta p)}{\kappa}\right)=\frac{1}{\kappa} \sqrt{\frac{(p \dot{x})}{\lambda}} .
$$

We find the following solutions,

$$
p_{\mu}=\frac{\dot{x}_{\mu}}{\lambda} ; \quad \lambda=\frac{\sqrt{\dot{x}^{2}}}{m}+\frac{1}{\kappa}(\eta \dot{x}) .
$$


Consistency of the computation is checked by noting that the solutions (85) satisfies the correct dispersion law we have imposed,

$$
p^{2}=\frac{(p \dot{x})}{\lambda}=m^{2}\left(1-\frac{(\eta p)}{\kappa}\right)^{2} .
$$

Finally we obtain the cherished Nambu-Goto Lagrangian for the $\kappa$-particle:

$$
L=\frac{m \sqrt{\dot{x}^{2}}}{\left(1+\frac{m(\eta \dot{x}}{\kappa \sqrt{\dot{x}^{2}}}\right)}\left(1+\frac{m}{\kappa}(\eta \dot{x})\left(\frac{(x \dot{x})}{\sqrt{\dot{x}^{2}}}\right)^{\cdot}\right) .
$$

Notice that (87) is a higher derivative Lagrangian. Hamiltonian analysis of it will yield the $\kappa$-NC phase space algebra.

The equation of motion $\dot{p}_{\mu}=0$ in (83) is very important. It is the analogue of the free particle equation of motion in normal spacetime (Newton's law) showing that momentum is conserved for the free $\kappa$-particle as well. We have already exploited this condition in Section III.

\section{VII: Quantization}

In this brief section we will try to argue through a few applications that the canonical coordinate approach is very convenient for quantization of the $\kappa$-particle. We recall from (37) the mapping between $\mathrm{NC} \kappa$-coordinates $\left(x_{\mu}, p_{\mu}\right)$ and canonical coordinates $\left(X_{\mu}, P_{\mu}\right)$ :

$$
x_{\mu}=X_{\mu}\left(1+\frac{(\eta P)}{\kappa}\right) ; \quad p_{\mu}=\frac{P_{\mu}}{\left(1+\frac{(\eta P)}{\kappa}\right)} .
$$

It is straightforward to elevate the classical Poisson brackets in $\left(X_{\mu}, P_{\mu}\right)$ to quantum commutation relations,

$$
\left[P_{\mu}, X_{\nu}\right]=i g_{\mu \nu} ;\left[X_{\mu}, X_{\nu}\right]=\left[P_{\mu}, P_{\nu}\right]=0
$$

In quantum theory the mapping has to be between operators,

$$
\hat{x}_{\mu}=\hat{X}_{\mu}\left(1+\frac{\hat{P}_{0}}{\kappa}\right) ; \quad \hat{p}_{\mu}=\frac{\hat{P}_{\mu}}{\left(1+\frac{\hat{P}_{0}}{\kappa}\right)} .
$$

However, notice that only the mapping between $x_{0} \equiv t$ and $X_{0} \equiv T$ needs operator ordering. Clearly there is no operator ordering ambiguity in non-relativistic quantum mechanical problems and since $P_{0}$ commutes with all the relevant variables $\vec{P}$ and $\vec{X}$ and one can replace $P_{0}$ by a number $m$, the particle mass in the non-relativistic limit. Hence the mapping becomes,

$$
\hat{x}_{i}=\hat{X}_{i}\left(1+\frac{(m)}{\kappa}\right) ; \quad \hat{p}_{\mu}=\frac{\hat{P}_{\mu}}{\left(1+\frac{m}{\kappa}\right)} .
$$

The advantage is that the operators $\hat{P}_{i}$ and $X_{i}$ can be treated in the conventional way.

Let us use the above ideas in the simplest problem, i.e. (non-relativistic) harmonic oscillator in $\kappa$-spacetime. The Hamiltonian is,

$$
H=\frac{\vec{p}^{2}}{2 m}+\frac{K}{2} x^{2}
$$




$$
=\frac{\vec{P}^{2}}{2 m\left(1+\frac{(m)}{\kappa}\right)^{2}}+\frac{K}{2}\left(1+\frac{(m)}{\kappa}\right)^{2} X^{2} \equiv \frac{\vec{P}^{2}}{2 \tilde{m}}+\frac{\tilde{K}}{2} x^{2} .
$$

This shows that the frequency,

$$
\omega=\sqrt{\frac{\tilde{K}}{\tilde{m}}}=\sqrt{\frac{K}{m}}
$$

and hence energy levels will remain unchanged.

We can consider the Hydrogen atom problem where the Hamiltonian is,

$$
\begin{gathered}
H=\frac{\vec{p}^{2}}{2 m}-\frac{Z e^{2}}{r} \\
=\frac{\vec{P}^{2}}{2 m\left(1+\frac{(m)}{\kappa}\right)^{2}}-\frac{Z e^{2}}{R\left(1+\frac{m}{\kappa}\right)} \equiv \frac{\vec{P}^{2}}{2 \tilde{m}}-\frac{Z \tilde{e}^{2}}{R} .
\end{gathered}
$$

Once again there is no change in the energy levels. This is actually obvious since the only effect of $\kappa$ in non-relativistic physics turns out to be a numerical scaling of the operators which is not observable.

\section{VIII: Conclusion and Outlook}

Let us summarize our work. We have concentrated on a particular Doubly Special Relativity model that has an underlying novel form of $\kappa$-Minkowski Noncommutative phase space structure along with a Magueijo-Smolin form of modified dispersion law. The novel and attractive feature of our work is the construction of (invertible) mapping between the Noncommutative $\kappa$-Minkowski phase space and a completely canonical phase space the latter obeying normal Poisson brackets. We further show the the canonical degrees of freedom transform under Special Theory Lorentz transformations whereas the original physical variables transform under $\kappa$-extended Lorentz transformations. Following this approach we have developed the complete structure of $\kappa$-deformation of the symmetry generators. These modified generators obey an un-deformed algebra, keep the $\kappa$-noncommutative phase space algebra stable under symmetry operations but induce deformed transformations on the phase space variables. Furthermore, the canonical construction simplifies the study of particle behavior in this particular DSR framework tremendously since the existing results of Special Theory directly applicable in the artificial canonical space. But from the explicit relation we have provided, these results can be mapped to the physical $\kappa$-Minkowski phase space, the latter having a much more complex Noncommutative Hamiltonian structure and generalized Lorentz transformations. Using our scheme, the free $\kappa$-particle action developed here can be extended to include interactions in a straightforward way. An outcome of our analysis is that $\kappa$-effects will probably not be visible in non-relativistic quantum systems.

As the next step in our programme we plan to investigate two problems:

(i) Behavior of $\kappa$-particle in the presence of interactions. We expect kinematical relations will change as we have indicated. We will introduce interaction term in the free particle Lagrangian in the canonical space since in canonical coordinates the interaction terms will have normal relativistic structure. Subsequently they can be mapped to $\kappa$-phase space coordinates and the dynamics can be studied. 
(ii) Construction of field theory in $\kappa$-NC spacetime. This is a very important issue and requires immediate attention. Our aim is to exploit the canonical approach once again. We intend to consider the field map $\varphi(x) \Rightarrow \phi(X)$. Notice that field theoretic Poisson brackets among $\varphi(x)$ and its momentum field will be non-canonical, (induced by $(x, p)-\kappa$-NC algebra), but brackets among $\phi(X)$ and its conjugate momentum field will be canonical. In this way it will be possible to map a field theory in $\kappa$-NC spacetime to a field theory in normal spacetime with normal fields. This construction should be compared with the (to be constructed) conventional *-product formulation of NC field theory.

Acknowledgements: We are grateful to Professor S.Mignemi for informing us about [13]. Also it is a pleasure to thank Professor S.Meljanac for a number of helpful correspondences. 


\section{References}

[1] G.Amelino-Camelia and T.Piran, Phys.Rev. D64 (2001)036005 (astro-ph/0008107).

[2] G.Amelino-Camelia, Nature 418 34(2002); Phys.Lett.B 510 (2001)255; Int.J.Mod.Phys. A11 (2002)35; For a review on DSR, see J.Kowalski-Glikman, hep-th/0405273.

[3] The earliest model for NC spacetime and physics in it is by H.S.Snyder, Phys.Rev. 71 (1947)68.

[4] Recently NC spacetime physics has become popular after its connection to low energy String physics was discovered by N.Seiberg and E.Witten, JHEP 9909(1999)032. For reviews see for example M.R.Douglas and N.A.Nekrasov, Rev.Mod.Phys. 73(2001)977; R.J.Szabo, Phys.Rept. 378 (2003) 207 (hep-th/0109162).

[5] C.Rovelli and L.Smolin, Nucl.Phys. B442 (1995)593; Erratum: ibid 456 (1995)734; For a recent review on quantum gravity phenomenology, see G.Amelino-Camelia, Mod.Phys.Lett. A17 (2002) 899 (gr-qc/0204051).

[6] S.Doplicher, K.Fredenhagen and J.E.Roberts, Phys.Lett. B331 39(1994).

[7] A.Kempf, G.Mangano and R.B.Mann, Phys.Rev.D 52 (1995)1108.

[8] J.Lukierski, A.Nowicki, H.Ruegg and V.N.Tolstoy, Phys.Lett. B264 (1991)331, S.Majid and H.Ruegg, Phys.Lett. B334 (1994)348; J.Lukierski, H.Ruegg, W.J.Zakrzewski, Annals Phys. 243 (1995)90.

[9] J.Magueijo and L.Smolin, Phys.Rev.Lett. 88 (2002)190403; Phys.Rev. D67 (2003)044017.

[10] J.Kowalski-Glikman and S.Nowak, Phys.Lett.B 539 (2002)126 (hep-th/0203040); Class.Quant.Grav. 20 (2003)4799 (hep-th/0304101); J.M.Romero and A.Zamora, Phys.Rev. D70 105006(2004).

[11] S.Ghosh, Phys.Lett. B648 (2007)262 (hep-th/0602009); Phys.Rev. D74 (2006) 084019 (hep-th/0608206).

[12] A.Granik, hep-th/0207113.

[13] S.Mignemi, Phys.Rev. D68 (2003) 065029 (gr-qc/0304029).

[14] A.A.Deriglazov, JHEP 0303 (2003) 021 (hep-th/0211105); A. Pinzul, A. Stern, Phys.Lett. B593 (2004) 279 (hep-th/0402220); S.Ghosh, Phys.Lett.B 623 (2005)251; S.Ghosh and P.Pal, Phys.Lett.B 618 (2005)243; F.Girelli, T.Konopka, J.Kowalski-Glikman and E.R.Livine, Phys.Rev. D73 (2006) (hep-th/0512107); R.Banerjee, S.Kulkarni and S.Samanta, JHEP 0605 (2006) 077 (hep-th/0602151); L.Freidel, F.Girelli and E.R.Livine, hep-th/0701113.

[15] N.R.Bruno, G.Amelino-Camelia and J.Kowalski-Glikman, Phys.Lett. B522 (2001) 133 (hep-th/0107039). 
[16] D.Kimberly, J.Magueijo and J.Medeiros, Phys.Rev. D70 (2004) 084007 (gr-qc/0303067).

[17] M.Chaichian, P.P.Kulish, K.Nishijima and A.Tureanu, Phys.Lett. B604 (2004)98 (hep-th/0408069); M.Chaichian, P.Presnajder and A.Tureanu, Phys.Rev.Lett. 94 (2005) 151602 (hep-th/0409096).

[18] P.Kosinski, J.Lukierski and P.Maslanka, hep-th/0009120; W.Dimitrijevic et.al., Eur.Phys.J. C31 (2003) 129 (hep-th/0307149); S.Meljanac and M.Stojic, Eur.Phys.J. C47 (2006) 531 (hep-th/0605133); S.Meljanac, S.Kresic-Juric and M.Stojic, hep-th/0702215.

[19] S.Ghosh, in preparation.

[20] A.A.Deriglazov and B.F.Rizzuti, Phys.Rev. D71 (2005) 123515, (hep-th/0410087).

[21] S.Hossenfelder, hep-th/0702016.

[22] M.Arzano and A.Marciano, hep-th/0701268.

[23] F.Girelli, S.Liberati and L.Sindoni, gr-qc/0611024; S.Hossenfelder, hep-th/0612167.

[24] P.A.M.Dirac, Lectures on Quantum Mechanics, Yeshiva University Press, New York, 1964. 